\title{
Satellite Panicum Mosaic Virus Capsid Protein Elicits Symptoms on a Nonhost Plant and Interferes with a Suppressor of Virus-Induced Gene Silencing
}

\author{
Wenping Qiu ${ }^{1}$ and Karen-Beth G. Scholthof ${ }^{2}$ \\ ${ }^{1}$ Department of Fruit Science, Southwest Missouri State University, Mountain Grove 65711, U.S.A.; ${ }^{2}$ Department of Plant \\ Pathology and Microbiology, 2132 TAMU, Texas A\&M University, College Station 77843, U.S.A.
}

Submitted 19 June 2003. Accepted 24 October 2003.

\begin{abstract}
The capsid protein (CP) of satellite panicum mosaic virus (SPMV) has been implicated as a pathogenicity factor, inducing severe chlorosis on millet plants co-infected with SPMV and its helper virus, Panicum mosaic virus (PMV). In this study, we tested the effects of SPMV CP on Nicotiana benthamiana, a plant that does not support PMV+SPMV infections. SPMV CP expressed from a Potato virus $X$ (PVX) gene vector elicited necrotic lesions on $N$. benthamiana. Pathogenicity factors often have the additional feature of acting as suppressors of gene silencing; therefore, several assays were developed to test if SPMV $C P$ could act in such a capacity. The results showed that SPMV CP failed to act as a suppressor of posttranscriptional gene silencing when such tests were performed with transgenic $N$. benthamiana plants silenced for green fluorescent protein (GFP) expression by agroinfiltration or plant virus vectors. However SPMV CP expressed from the PVX gene vector did interfere with suppressor activity associated with PVX p25. This included a rebounded level of GFP silencing along the vascular tissues, including the veins on upper noninoculated leaves. Therefore, the roles of the SPMV CP now include encapsidation of the SPMV RNA, activity as a pathogenicity factor in both host and nonhost plants, and the enigmatic feature of interfering with suppression of gene silencing.
\end{abstract}

A group of viral proteins that block either posttranscriptional gene silencing (PTGS) or virus-induced gene silencing (VIGS) pathways in host plants have been identified and are referred to as viral suppressors (Anandalakshmi et al. 1998; Beclin et al. 1998; Brigneti et al. 1998; Kasschau and Carrington 1998; Qiu et al. 2002; Voinnet et al. 1999). Prior to characterization of viral suppressors, some of these proteins were shown to induce severe viral symptoms (Ding et al. 1996; Scholthof et al. 1995a) or have a role in synergistic interactions with an unrelated virus (Pruss et al. 1997). That both activities can be attributed to a protein suggests an intrinsic relationship between viral pathogenesis and the suppression of gene silencing. From this, it follows that pathogenesis factors should be valuable reagents to dissect and define biochemical pathways in the plant that regulate silencing, suppression, or both.

Satellite panicum mosaic virus (SPMV) is an 824-nucleotide (nt) plus-sense, single-stranded RNA that is dependent on

Corresponding author: K.-B. G. Scholthof; Telephone: 979-845-8265; Fax: 979-845-6483; E-mail: kbgs@tamu.edu
Panicum mosaic virus (PMV) for replication and spread (Scholthof 1999b; Turina et al. 1998, 2000). In natural infections, SPMV capsid protein (CP) is used to encapsidate SPMV RNA and PMV satellite RNAs (Cabrera and Scholthof 1999; Desvoyes and Scholthof 2000; Qiu and Scholthof 2001b). Under experimental conditions, the SPMV CP coding region is not necessary for replication or spread of the SPMV RNA (Qiu and Scholthof 2000). Plants co-infected with PMV+SPMV display substantially more severe symptoms than those infected with PMV alone. In fact, SPMV exacerbates the normally mild PMV symptoms to induce stunting and a severe chlorotic mottle on millet plants. There is also a concomitant increase in PMV RNA and at least two PMV-encoded proteins, the 26-kDa CP and p8 (Scholthof 1999b). We have since determined that the SPMV CP is a crucial component for elicitation of symptoms on proso millet plants and, by reverse genetics and mutagenesis, defined the C-terminal half of the SPMV CP as a "chlorosis-inducing" domain (Qiu and Scholthof 2001b).

In an effort to understand the underlying mechanism of the SPMV CP-induced pathogenesis, SPMV CP was introduced into Nicotiana benthamiana, a plant that does not support replication or spread of PMV or PMV+SPMV. Two plant virusderived gene vectors, Tomato bushy stunt virus (TBSV) (Scholthof 1999a) and Potato virus X (PVX) (Chapman et al. 1992), and agroinfiltration were used for in planta delivery of SPMV CP. The expression of SPMV CP from the PVX gene vector $[\mathrm{PVX}-\mathrm{SPCP}(+)]$ induced a necrotic response restricted mainly along class II and III veins of $N$. benthamiana leaves. In transgenic $N$. benthamiana expressing the green fluorescent protein (GFP) gene (NbGFP), silencing was induced by a recombinant TBSV vector containing the same GFP gene. When SPMV CP was expressed from PVX-SPCP(+), vascular necrosis and partial silencing was maintained, especially along the class II and III veins. This was unexpected in that the PVX p25 gene encodes a protein that is a strong suppressor of gene silencing. Therefore, it appears that SPMV CP has the unusual ability to interfere with a well-characterized suppressor of VIGS.

\section{RESULTS}

SPMV CP induces symptoms on a nonhost plant when expressed from a PVX gene vector.

The SPMV CP coding region was cloned into PVX-E1S, a modified PVX viral vector, to create PVX-SPCP(+) and PVXSPCP(-), respectively (Fig. 1A). Systemic infections for each of the recombinant PVX viruses were confirmed on NbGFP 
A
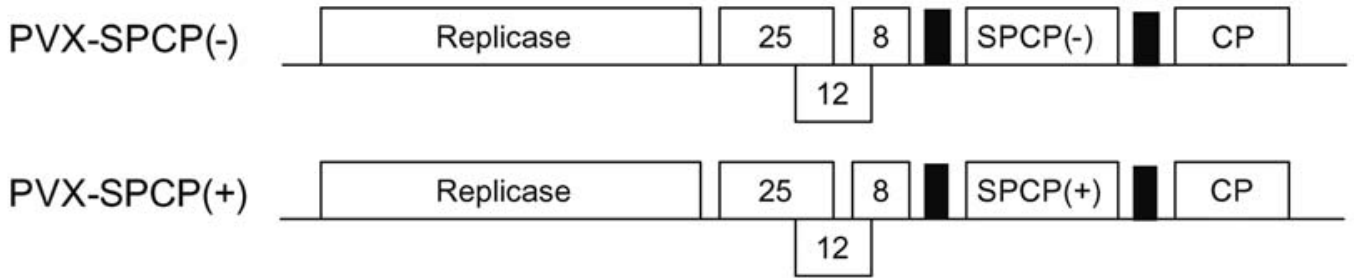

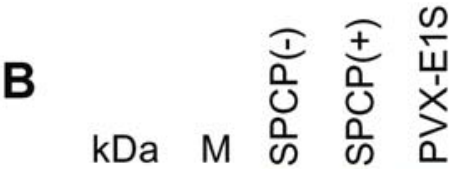

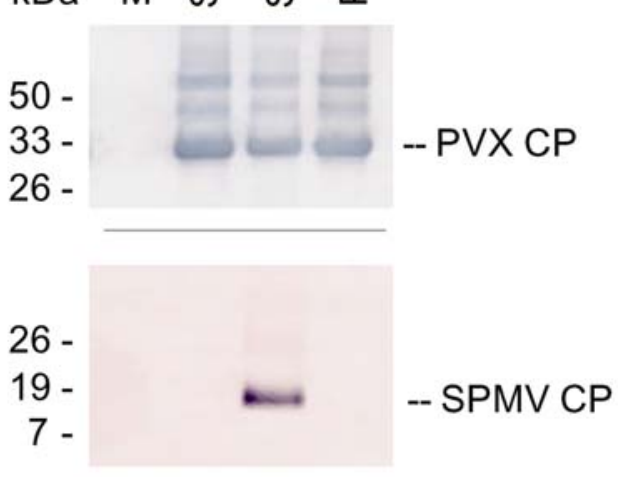

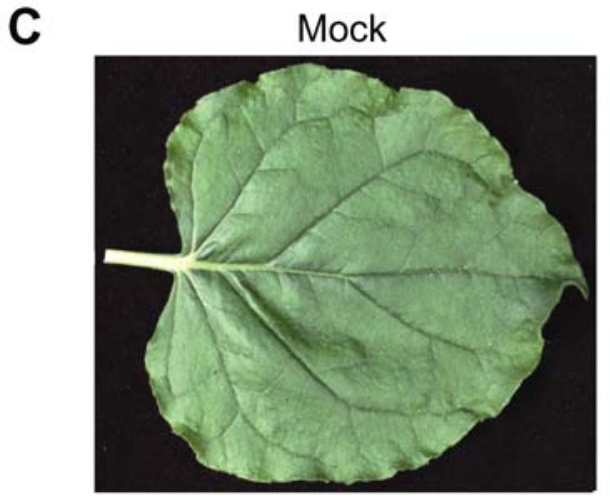

PVX-SPCP(+)

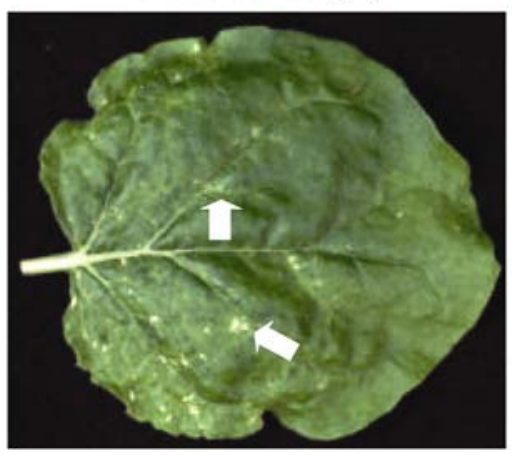

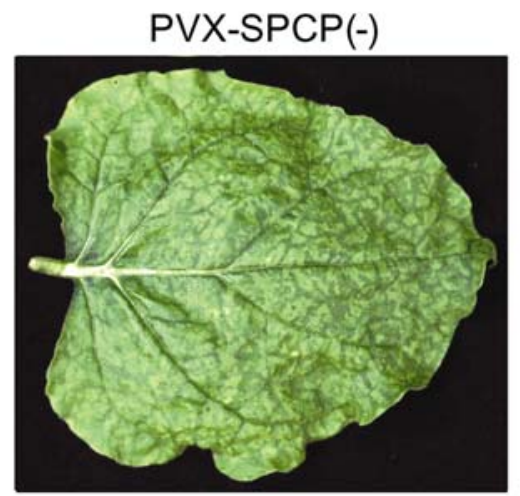

PVX-E1S

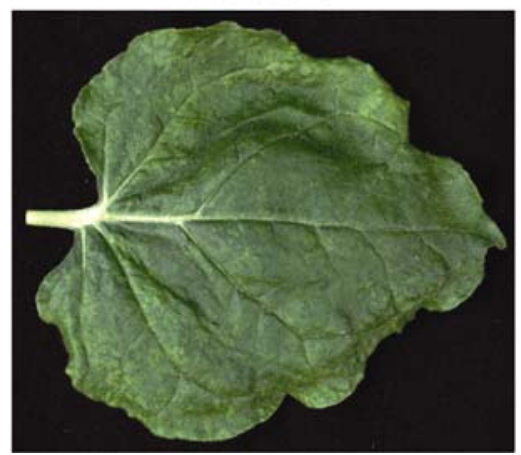

Fig. 1. Expression of satellite panicum mosaic virus (SPMV) capsid protein (CP) from a Potato virus $X$ (PVX) gene vector. A, Schematic illustration of the PVX vector and its derivatives. The drawings are not to scale. The transcription of the SPMV CP insert is under the control of a duplicated PVX CP promoter (filled bars). Open boxes denote open reading frames (ORFs) that encode proteins. The replicase, triple gene block (p25, p12, and p8) and CP ORFs on the PVX genome are indicated. Solid lines represent presumed untranslated sequences. B, Immunoblot assay of total proteins from upper, noninoculated leaves of green fluorescent protein (GFP) transgenic $N$. benthamiana (NbGFP) plants 21 days postinoculation (dpi) with transcripts of PVX-SPCP(-), PVXSPCP(+), or PVX-E1S. M indicates mock-inoculated plants. SPCP(+) and SPCP(-) refer to inoculation with PVX-SPCP(+) and PVX-SPCP(-) transcripts, with the SPMV CP in the plus or minus orientation, respectively. The top panel shows the presence of PVX CP in plants infected with the PVX vector, PVX-SPCP(+), and PVX-SPCP(-). The bottom panel shows that expression of the 17.5-kDa SPMV CP from the PVX gene vector occurs only from PVXSPCP(+)-infected plants, as predicted. The molecular weight protein standards (kDa) and location of PVX CP or SPMV CP is indicated alongside each panel. C, Symptoms on systemically infected leaves of NbGFP plants inoculated with transcripts of the PVX vector and its SPMV-CP chimeras, as labeled. The symptoms were recorded at 16 dpi. The white arrows point to necrotic patches predominantly along the stem, major vein, and class III veins on NbGFP leaves infected with PVX-SPCP(+). 
plants at 21 days postinoculation (dpi) with immunoassays specific for PVX CP expression (Fig. 1B, top panel). SPMV $\mathrm{CP}$ also was detected at relatively high levels in infected NbGFP plants inoculated with PVX-SPCP(+) (Fig. 1B, bottom panel). As anticipated, SPMV CP did not accumulate in plants inoculated with PVX-SPCP(-) and PVX-E1S.

Typical PVX-like mosaic symptoms were observed on NbGFP plants infected with PVX-SPCP(-) and PVX-E1S (Fig. 1C). Expression of SPMV CP from the PVX vector was positively correlated with appearance of necrosis on NbGFP plants. PVX-SPCP(+)-infected plants developed distinctive necrotic symptoms along the stem and the veins as well as a "patchy" necrosis on the leaves at 8 to $10 \mathrm{dpi}$ (Fig. 1C). This is in agreement with our previous observations that SPMV CP is a pathogenesis determinant in host plants and supports our premise that the satellite virus $\mathrm{CP}$ induces a distinguishable phenotype even when expressed in a nonhost plant, such as $N$. benthamiana.

\section{SPMV CP does not affect PTGS.}

To determine whether or not other biological roles were coupled with SPMV CP expression, NbGFP plants were inoculated with PVX-SPCP(+) and PVX-SPCP(-), and infiltrated 1 day later with Agro/GFP. In contrast with the previous systems, where viruses were inoculated onto plants following infiltration with Agro/GFP in order to identify viral suppressors, the assays developed in this study can investigate the potential effects of pre-expressed SPMV CP on gene silencing induced by agroinfiltration. These results suggested that PVXexpressed SPMV CP did not influence the agroinfiltrationmediated gene silencing (Table 1).

Considering the possibility that the inability of PVXexpressed SPMV CP to influence gene silencing may be due to a low expression level of SPMV CP from the PVX gene vector within $1 \mathrm{dpi}$, we performed the second experiments of agroinfiltration at $14 \mathrm{dpi}$ with $\mathrm{PVX}-\mathrm{SPCP}(+)$ and PVXSPCP(-), respectively. Immunoblot assays confirmed that SPMV CP was expressed in plants inoculated with PVX$\mathrm{SPCP}(+)$ (data not shown). Although there were variations of the number of plants showing systemic silencing of GFP genes in two independent tests on NbGFP plants that were infiltrated with Agro/GFP bacteria at 14 dpi of PVX recombinant viruses (Table 1), the observations indicated that PTGS occurred on all NbGFP plants infiltrated with Agro/GFP, independent of whether plants were inoculated previously with $\mathrm{PVX}$ SPCP(+), PVX-SPCP(-), or buffer.
SPMV CP does not suppress VIGS.

The influence of the SPMV CP on gene silencing was tested with DI-P (defective interfering RNA), a TBSV-derived DIgene vector carrying approximately $200 \mathrm{nt}$ of the $3^{\prime}$ proximal end of the GFP gene. It previously was determined that DI-P rapidly silences GFP gene expression in NbGFP plants, when co-infected with transcripts of a TBSV construct incapable of expressing the p19 gene (Qiu et al. 2002). In this case, pHST214 (Scholthof 1999a), a TBSV CP gene-replacement vector, was used to construct plasmids pTBSV-SPCP(+) and pTBSV$\operatorname{SPCP}(-)$, with the SPMV CP gene in plus- and minus-sense orientations, respectively (Fig. 2A). Transcripts from these vectors were used in co-inoculations with DI-P. When TBSV$\mathrm{SPCP}(+)$ or TBSV-SPCP(-) transcripts were inoculated onto NbGFP plants with DI-P, both TBSV genomic (g) and subgenomic (sg) RNAs were detected (Fig. 2B). SPMV CP also accumulated in TBSV-SPCP(+)-infected plants (Fig. 2C). Comparisons of the two assays reveal that DI-P consistently accumulated more abundantly as a mixed infection with TBSV-SPCP(-) than with TBSV-SPCP(+) (Fig. 2B). By extension, this suggests that the SPMV CP may stabilize the TBSV genomic RNA to retard further DI formation. What is more typical for TBSV-DIs is the observation made for the $\operatorname{SPCP}(-)$ experiment, with the DI resulting in significant reduction of its helper virus TBSV-SPCP(-) (Fig. 2B).

At 6 to 8 dpi, silencing of GFP mRNA in NbGFP plants was observed on leaves that were co-inoculated with DI-P and either TBSV-SPCP(+) or TBSV-SPCP(-). A DI-P-mediated dull red fluorescent ringspot pattern occurred in the presence of TBSV-SPCP( $(-)$ or TBSV-SPCP $(+)$ that was distinct from the blotchy red fluorescence pattern induced by transcripts of pTG or Agro/GFP (Qiu et al. 2002). At 10 dpi, the red fluorescence began to appear along the class II and III veins on the leaves immediately above the inoculated leaves. The red fluorescence gradually expanded to the newly emerging leaves and ultimately reached the entire plants at approximately $28 \mathrm{dpi}$ (data not shown). Corresponding to the typical VIGS-associated expansion of red fluorescence along the entire plant, GFP mRNAs was not detected, indicating that systemic silencing had occurred (Fig. 2D). It cannot be discounted completely that the inability of SPMV CP to suppress DI-induced gene silencing might be dose dependent (Qiu et al. 2002) or due to undetected effects, including regulation of SPMV CP expression from TBSV-SPCP(+) RNA. However, the fluorescence transition and the abolishment of GFP mRNA accumulation (Fig. 2D) indicate that DI-P efficiently initiated local and sys-

Table 1. Rate of systemic silencing of green fluorescent protein (GFP) gene in NbGFP plants inoculated with a Potato virus $X$ (PVX)-gene vector containing the satellite panicum mosaic virus (SPMV) capsid protein (CP) in either the plus or minus orientation, or mock inoculation, followed by agroinfiltration with $A g r o / G F P$ at 1 or 14 days postinoculation (dpi)

\begin{tabular}{|c|c|c|c|c|}
\hline \multirow[b]{2}{*}{ Agroinfiltration (dpi), inoculum ${ }^{a}$} & \multicolumn{4}{|c|}{ Days following agroinfiltration when GPF-gene silencing was observed } \\
\hline & 7 & 9 & 11 & 20 \\
\hline \multicolumn{5}{|l|}{$\overline{1^{\mathrm{b}}}$} \\
\hline PVX-SPCP(-) & $8 / 9$ & $8 / 9$ & $9 / 9$ & 9/9 \\
\hline PVX-SPCP(+) & $6 / 9$ & 9/9 & $9 / 9$ & 9/9 \\
\hline Mock & $4 / 9$ & $6 / 9$ & $6 / 9$ & 9/9 \\
\hline \multicolumn{5}{|l|}{$14^{\mathrm{c}}$} \\
\hline PVX-SPCP(-) & $1 / 6$ & $4 / 6$ & $5 / 6$ & $6 / 6$ \\
\hline PVX-SPCP(+) & $2 / 6$ & $4 / 6$ & $5 / 6$ & $6 / 6$ \\
\hline Mock & $3 / 6$ & $5 / 6$ & $6 / 6$ & $6 / 6$ \\
\hline
\end{tabular}

${ }^{a}$ Agroinfiltration with Agro/GFP at 1 or 14 dpi. An experiment consisted of three NbGFP plants that were infected with PVX-SPCP(+), PVX-SPCP(-), or mock-inoculated.

${ }^{\mathrm{b}}$ Numerator indicates the number of plants showing systemic silencing of GFP after agroinfiltration with Agro/GFP. At 1 dpi, all of the inoculated leaves, including Mock, were infiltrated with Agro/GFP. Visual monitoring of systemic silencing of GFP was recorded daily. The experiments were repeated three times, for a total of nine plants as tabulated.

${ }^{c}$ Numerator shows the number of plants showing systemic silencing of GFP following agroinfiltration with Agro/GFP at 14 dpi of transcripts. The numerator shows six plants; three plants per treatment, with the experiment repeated twice. 

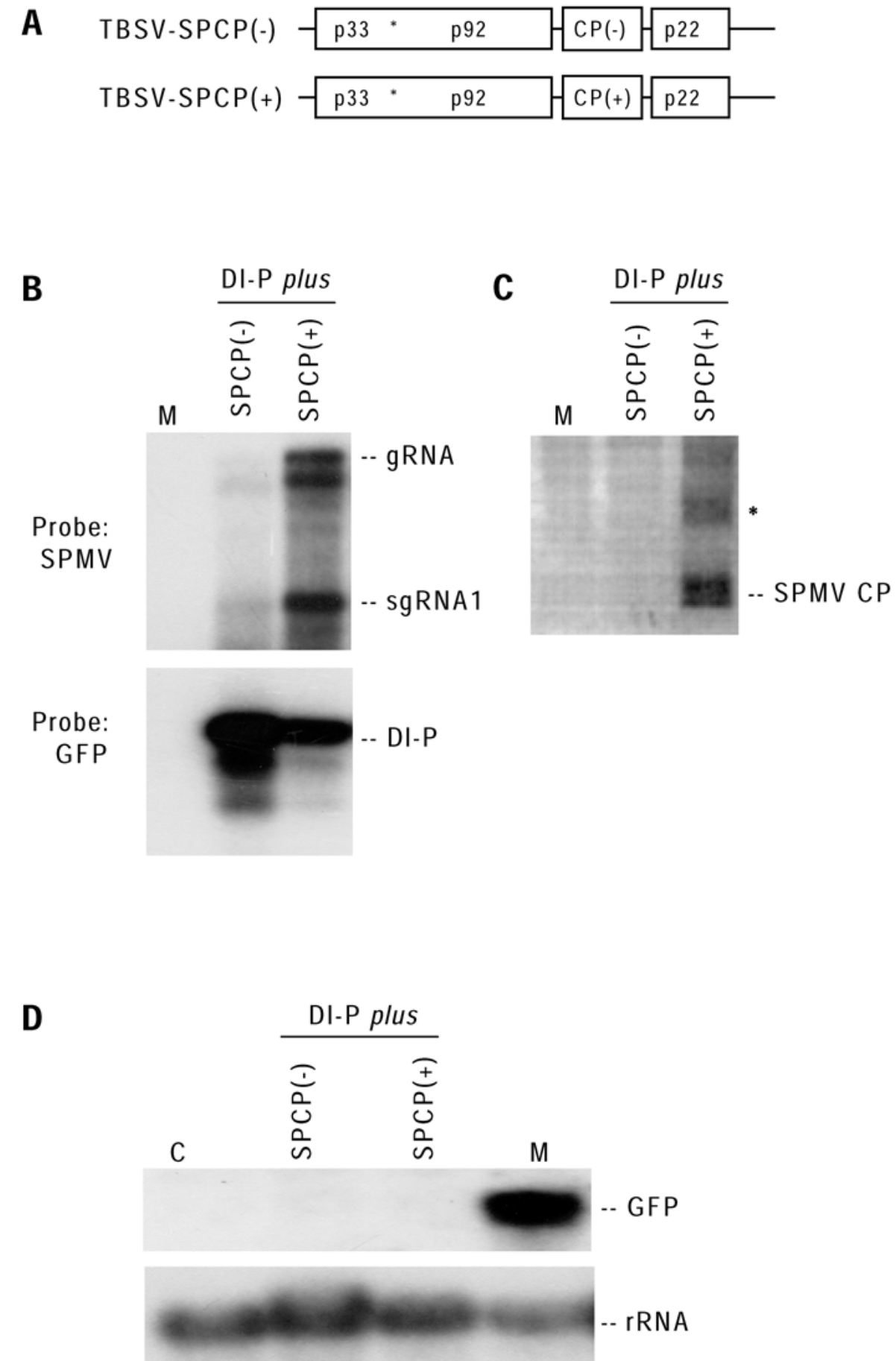

Fig. 2. Expression of satellite panicum mosaic virus (SPMV) capsid protein (CP) from a Tomato bushy stunt virus (TBSV) vector and analysis of the influence of SPMV CP on gene silencing through a co-delivery system on green fluorescent protein (GFP) transgenic $N$. benthamiana (NbGFP) plants. A, Schematic representation of two TBSV-derived constructs, TBSV-SPCP $(-)$ and TBSV-SPCP $(+)$, with the SPMV CP coding region in a minus or plus orientation, respectively. The drawings are not to scale. Open boxes denote open reading frames (ORFs) that encode proteins whose sizes are given in kDa inside the boxes. The asterisk (*) inside the TBSV genome indicates the position of an amber codon used for translational read-through of p33 to yield the p92 product. Both p33 and p92 are required for TBSV replication. P22 is the TBSV cell-to-cell movement protein. For the purposes of this study, TBSV p19 gene expression was abolished, because it is a known suppressor of gene silencing. Solid lines represent presumed untranslated sequences on the TBSV genome. B, RNA-blot assay of total RNA isolated from NbGFP plants that were co-inoculated with a 1:2 ratio of DI-P plus TBSV-SPCP(-) or DI-P plus TBSV-SPCP(+). DI-P is a TBSV defective interfering RNA containing the $3^{\prime}$ end of the GFP gene (Qiu et al. 2002). M indicates mock-inoculated plants. On the figures, SPCP(+) or SPCP(-) refer to plants inoculated with TBSV-SPMV(+) or TBSV-SPMV(-), respectively. The membrane was hybridized with a ${ }^{32} \mathrm{P}$-dCTP-labeled, GFP-specific DNA probe to detect DI-P RNA and subsequently hybridized with an SPMV-specific probe to assay for the presence of the SPMV fragment on the chimeric TBSV-SPCP(+) and TBSV-SPCP(-) viruses. Total RNA was extracted from the inoculated leaves at 6 days postinoculation (dpi). C, Immunoblot assay of total proteins from plants inoculated as described for panel B. Total proteins were extracted from inoculated leaves at 6 dpi. An SPMV CP-specific polyclonal antibody was used to detect the 17.5-kDa SPMV CP (arrow). The band indicated by an asterisk likely represents a dimer of the SPMV CP. D, RNA blot assay for GFP mRNA in either silenced or nonsilenced tissues from NbGFP plants inoculated as described for panel B. An rRNA-specific probe also was used to indicate the relative amounts of RNA for each sample. Total RNA was extracted from systemically silenced NbGFP leaves at 28 dpi from a mixed infection of DI-P plus TBSV-SPCP(-) or DI-P plus TBSV-SPCP(+). A mock (M) infiltration reveals the GFP expression in NbGFP plants and the control (C) lane shows GFP-mediated gene silencing at 23 days postinfiltration with Agro/GFP. 
temic GFP gene silencing even in the presence of the SPMV $\mathrm{CP}$ expressed from the TBSV vector. Therefore, SPMV CP does not interfere with TBSV DI-mediated VIGS.

\section{SPMV CP interferes with PVX-p25 suppressor activity after the induction of VIGS.}

In these experiments, a recombinant TBSV vector (pTG) with an inactivated p19 gene and expressing the GFP gene substituted for the TBSV CP gene was used as the silencing inducer on NbGFP plants (Qiu et al. 2002). After the pTG transcripts were combined with either PVX-E1S or PVX$\mathrm{SPCP}(+)$ in a 1:1 ratio and inoculated onto NbGFP plants, the presence of PVX, pTG, and SPMV CP was confirmed by RNA and immunoblot assays (Fig. 3A and B). RNA blots hybridized with a GFP-specific probe showed that pTG RNA accumulated in the inoculated leaves infected with the transcripts of pTG alone or when co-inoculated with PVX-SPCP $(+)$ or PVX-E1S (Fig. 3A). As expected, PVX CP accumulated in plants infected with PVX-SPCP(+) or PVX-E1S, but not in plants infected with only pTG (Fig. 3B, top panel). SPMV CP readily accumulated in the plants that were co-inoculated with pTG and PVX-SPCP(+) (Fig. 3B, bottom panel). These results confirmed that both the SPMV CP and the gene-silencing inducer pTG were present in the infected tissues.

At 8 to $10 \mathrm{dpi}$, red fluorescence (local silencing) was observed on the inoculated leaves of all plants inoculated with pTG alone or as mixed infections with PVX-E1S or PVXSPCP(+) (Fig. 3C). Except for mock inoculation, a characteristic patchy red pattern developed on all inoculated leaves, which is typical of pTG-mediated GFP gene silencing (Qiu et al. 2002). However, at $10 \mathrm{dpi}$, red fluorescence was not observed on the upper noninoculated leaves of plants infected with pTG plus PVX-E1S or pTG alone (Fig. 3C, middle and bottom panels), which we interpreted as a lack of induction of systemic gene silencing. In contrast, by $10 \mathrm{dpi}$, NbGFP plants co-infected with pTG and PVX-SPCP $(+)$ reproducibly developed red fluorescence, typically along the class III veins of leaves immediately above the inoculated leaves (Fig. 3C, top panel). Class II veins of newly emerging leaves on these plants also frequently displayed a red fluorescent vein-banding pattern (Fig. 3C, top panel). At $11 \mathrm{dpi}$, red fluorescence also began to appear on the upper leaves of plants inoculated with pTG alone. It was observed in three independent experiments that vein-banding red fluorescence appeared 1 day earlier on the plants that were co-inoculated with pTG plus PVX$\operatorname{SPCP}(+)$ than on those that were inoculated with pTG alone (data not shown).

At 16 dpi, green fluorescence was maintained on the upper noninoculated leaves of plants infected with pTG+PVX-E1S (Fig. 3C, middle panel), suggesting that pTG-mediated silencing was suppressed by co-infection with PVX-EIS. In contrast, plants inoculated with pTG alone were exhibiting red fluorescence, mainly along the class III veins (Fig. 3C, bottom panel). This class III vein-banding pattern of red fluorescence also was observed for pTG plus PVX-SPCP $(+)$ infections, with a subsequent rapid expansion to class I veins by 16 dpi (Fig. 3C, top panel). Therefore, it appears that the SPMV-CP counteracts the PVX suppressor, p25.

Furthermore, and of great interest, was the observation that SPMV CP accumulation caused an intermediate GFP-expression phenotype that correlated with relative GFP accumulation. NbGFP plants expressing SPMV CP from the PVX vector had detectable levels of GFP mRNA (Fig. 3A), although much less than those of PVX-E1S-infected plants, even though both vectors encode the PVX p25 (suppressor) open reading frame (ORF). In pTG-infected plants, silencing was complete, and GFP RNA was not detected. Observations of the leaves of
pTG-inoculated NbGFP plants at 16 dpi showed that green fluorescence was much less abundant in the leaves of plants infected with pTG plus PVX-SPCP(+) compared with infections of pTG plus PVX-E1S, or for mock-inoculated NbGFP plants (Fig. 3C). These observations confirmed that the relative level of red fluorescence was correlated positively with the degradation of GFP mRNA in the silenced tissues (Fig. 3A and $\mathrm{C}$ ).

In summary, we find that i) SPMV CP is biologically active as a pathogenicity factor on a nonhost plant, ii) SPMV CP is not a suppressor of VIGS or PTGS, and iii) SPMV CP has the novel feature of interfering with the activity of a well-characterized suppressor of VIGS. The observations suggest that virus pathogenicity and suppression are independent features of SPMV CP and it is likely that the multiple mechanistic roles of this protein can be dissected and defined.

\section{DISCUSSION}

\section{SPMV CP as a pathogenesis factor.}

Our previous studies demonstrated that SPMV replication and spread by its helper virus PMV was not impaired when the SPMV CP coding region was deleted from the genomic RNA of SPMV (Qiu and Scholthof 2000). This feature also has been reported for other satellite viruses (Bringloe et al. 1998; Routh et al. 1997). In mixed infections of PMV+SPMV on millet, SPMV CP has several functions, including encapsidation of the satellite genomic RNA (Desvoyes and Scholthof 2000), the involvement in defective RNA accumulation, and the induction of severe chlorosis on host plants (Qiu and Scholthof 2001a,b; Scholthof 1999b). The present study shows that SPMV CP, when it was expressed by a PVX viral vector, was able to induce necrotic symptoms on $N$. benthamiana, a nonhost for PMV and SPMV infections, but a very susceptible host plant to a majority of plant viruses. The symptoms are distinct from the typical generalized mosaic associated with infections of PVX (Fig. 1) or PVX-GFP vectors (Toth et al. 2001). The pathogenic effects of SPMV CP most likely mirror its role as a pathogenesis factor in host millet plants that support PMV+SPMV infections. Also, the ability to induce these host and nonhost symptoms maps to the approximately $10-\mathrm{kDa} \mathrm{C}$ terminal portion of the $17.5-\mathrm{kDa}$ SPMV CP gene (data not shown). This finding reinforces and extends the previous conclusion that the SPMV CP is a symptom determinant (Qiu and Scholthof 2001b).

It is important to note that the SPMV CP-associated necrotic symptoms along the small veins of $N$. benthamiana plants infected with PVX-SPCP(+) did not resemble the original syndrome of severe chlorosis in the millet host plants. The distinctiveness of the pathogenesis processes triggered by a viral protein in a host plant versus in a nonhost plant previously was shown with Caulimovirus gene VI proteins (Goldberg et al. 1991; Schoelz et al. 1991). When expressed in a transgenic host plant, Caulimovirus gene VI proteins did not generate visible symptoms, but induced a strong mosaic or severe necrosis in transgenic nonhost plants (Goldberg et al. 1991). Similarly, the P1 protein of Rice yellow mottle virus (RYMV) is a pathogenesis determinant on rice (Bonneau et al. 1998) and also induces severe necrotic symptoms on $N$. benthamiana, a nonhost plant, when expressed from a PVX vector (Voinnet et al. 1999). Based on our findings, the evidence supports a biologically significant role for SPMV CP. Therefore, the SPMV CP, RYMV P1, and the Caulimovirus gene VI proteins each share an ability to induce a cascade of molecular events in host and nonhost plants that result in distinctive phenotypes. 

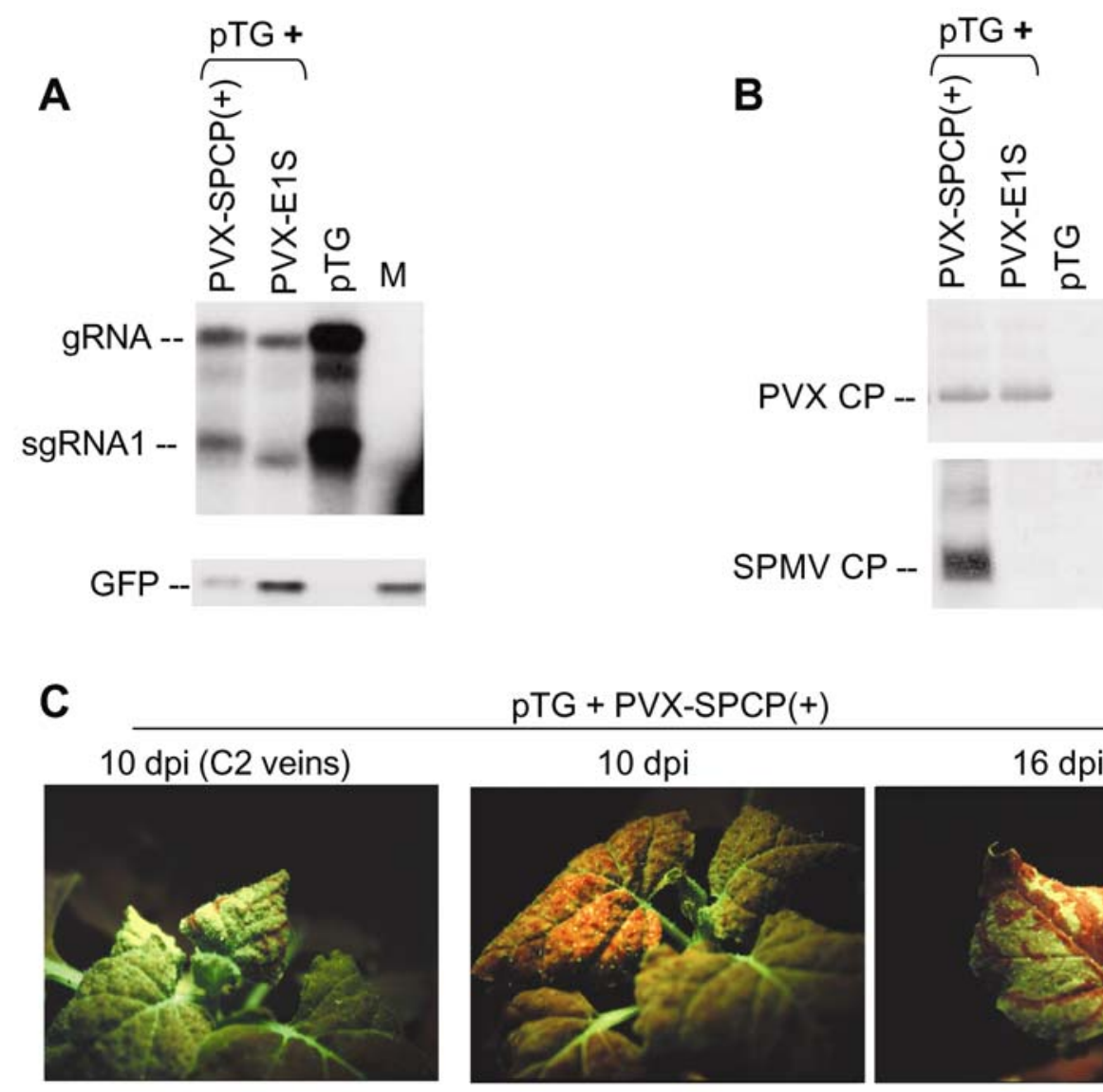

pTG + PVX-SPCP $(+)$
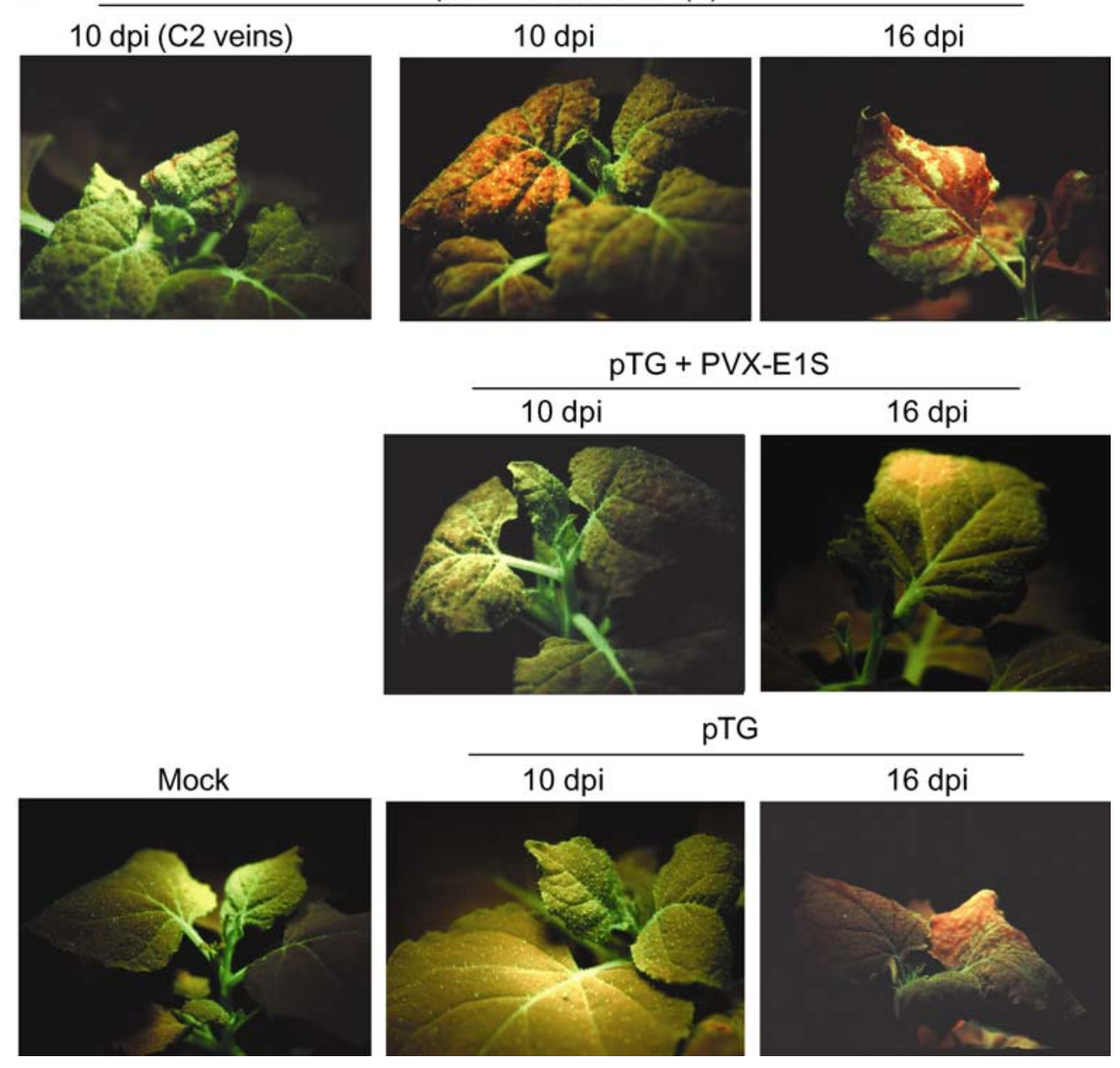

Fig. 3. A system for analyzing the influence of satellite panicum mosaic virus (SPMV) capsid protein (CP) on gene silencing through the co-delivery of SPMV CP from a Potato virus $X$ (PVX) viral vector and a gene silencing inducer (pTG) onto green fluorescent protein (GFP) gene (NbGFP) plants. The construct pTG is a Tomato bushy stunt virus (TBSV)-based gene vector, expressing the GFP gene. Co-inoculations of NbGFP plants with pTG and PVXSPCP(+) or pTG and PVX-E1S transcripts were in a 1:1 ratio. A, The top panel represents an RNA blot showing the accumulation of pTG RNA from total RNA isolated from inoculated leaves at 12 days postinoculation (dpi). The bottom panel shows GFP mRNA accumulation (to monitor pTG-induced gene silencing) in the upper, noninoculated leaves of NbGFP plants at 16 dpi. These blots also were hybridized with rRNA specific probes (not shown). B, Immunoblot analysis for the expression of the PVX CP (top panel) and the SPMV CP (bottom panel). Total plant proteins were extracted from the systemically infected leaves at $16 \mathrm{dpi}$. C, GFP transgene silencing patterns, as indicated by the progressive transition of fluorescence, following induction by pTG alone or as co-inoculations of pTG and PVX-E1S or pTG and PVX-SPCP $(+)$ on NbGFP plants. Systemic transgene silencing of the upper noninoculated leaves was recorded at 10 and $16 \mathrm{dpi}$, respectively. Gene silencing appeared at $10 \mathrm{dpi}$ along the class II veins (indicated by $\mathrm{C} 2$ ) of the newly emerging leaf (top panel, left-most image) of plants co-inoculated with pTG and PVX-SPCP(+), but not on plants with the other combinations of RNA transcripts (middle and bottom panels). The striking green fluorescence from NbGFP plants is shown for a mock-inoculated plant. 
Virus-induced necrosis is the outcome of complex signaling pathways in plant cells in response to specific (toxic) viral proteins (Culver et al. 1991; Gilchrist 1998; Greenberg 1997; Heath 2000). Massive necrosis and ultimate collapse of $N$. benthamiana plants is caused by TBSV P19, Tobacco etch virus $\mathrm{P} 1 / \mathrm{HC}$-Pro, and RYMV P1 expression from the PVX vector (Pruss et al. 1997; Scholthof et al. 1995a; Voinnet et al. 1999). In contrast, SPMV CP-induced necrosis is not lethal and remains confined to the vascular tissues along the stem and, sporadically, along the class II and III veins of the upper, noninoculated leaves (Fig. 1C). This suggests that the vascular tissue-restricted necrosis induced in the presence of SPMV CP reflects a general cell death process as a nonhost response to a "foreign" viral protein. This phenomenon also may be dosedependent because the transient expression of SPMV CP through agroinfiltration or from the TBSV vector failed to induce visible symptoms on $N$. benthamiana. However, movement of TBSV (and, therefore, SPMV CP) is restricted because the vector does not express the TBSV capsid and p19 proteins. It also is possible that the vascular tissue-restricted necrosis is a result of an interaction between the SPMV CP and PVX proteins.

\section{SPMV CP does not affect VIGS and PTGS.}

Gene silencing was induced in NbGFP plants that were coinoculated with pTG plus PVX-SPCP(+) or pTG alone (Fig. 3 ). These experiments confirmed that the TBSV p19 protein, a strong suppressor of VIGS (Qiu et al. 2002), was inactivated and that SPMV CP does not interfere with silencing. Systemic silencing was blocked when PVX-E1S was co-inoculated with pTG on NbGFP plants, confirming earlier observations that PVX p25 is a suppressor of VIGS (Voinnet et al. 2000). Therefore, PVX was used as a vector to determine whether or not other biological effects could be linked to SPMV CP expression.

SPMV CP expression from PVX had clear contrary effects on the p25 suppressor. This suggests that SPMV CP may counter an obstruction of mobile silencing signals imposed by the PVX-encoded p25 protein. This, in turn, would permit the transport of the silencing signal. This agrees with our observation that pTG-mediated silencing was accelerated approximately 1 day in the presence of the SPMV CP compared with the pTG infection alone and the transition to red fluorescence frequently was restricted to class II and III veins on the newly emerging leaves (Fig. 3C). The vein-restricted silencing of the GFP gene was confirmed further by RNA blots that showed GFP mRNA accumulated in leaf tissues of (partially) silenced plants following co-inoculation with pTG and PVX-SPCP(+) (Fig. 3A).

Interestingly, the gene-silencing pattern in the presence of the SPMV CP was the same as the necrosis response in that both occurred along vascular tissues in plants inoculated with PVX-SPCP(+) (Fig. 1C). This may suggest that the SPMV $\mathrm{CP}$-mediated vascular-restricted necrosis accelerated the silencing signal spread. Alternatively, or in addition, the SPMV CP may bind to and either assist with transport of the small RNAs or protect them from the host RNases in the signal transport route. This acceleration phenomenon was not observed in the plants that were inoculated with $\operatorname{PVX}-\operatorname{SPCP}(+)$ and subsequently infiltrated with Agro/GFP (Table 1). Thus, it appears that SPMV CP is an accelerant of VIGS, but not of agroinoculation-mediated gene silencing. How does this relate to the role of the SPMV CP in wild-type infections with PMV? Could such processes be associated with the origins of satellite RNA? Clearly, SPMV CP is providing some interesting hints of the origins and modes of action of virus proteins that have dual roles in pathogenesis and suppression.
The question remains as to whether the SPMV CP alone is affecting the biological activity of p25 or if the SPMV RNA has a viable role in this observed effect. At this time, the preponderance of evidence suggests that the SPMV CP is the primary "actor" in this event (Qiu and Scholthof 2000, 2001b). Unfortunately, nontranslatable controls in this system are not suitable because the inframe downstream start codons on the SPMV CP ORF are active even if the authentic start codon (nucleotide 88) is disrupted and stop codons are introduced immediately downstream of the start codon (Qiu and Scholthof 2001b). Therefore, the question remains: is it the $\mathrm{CP}$ or the $\mathrm{CP}$ RNA? Using SPMV $\mathrm{CP}(-)$ as a control, we found that the minus-sense SPMV CP RNA does not induce the patchy leaf necrosis or vein necrosis on $N$. benthamiana that was observed for the SPMV-CP(+) construct expressed from the respective PVX vectors (Fig. 1). Similarly, the minus-sense CP RNA did not interfere with TBSV DI accumulation or silencing effects when TBSV-SPCP(-) was co-inoculated with DI-P (Fig. 2). From this, we can surmise that the effects are specific to SPMV CP, but we cannot completely rule out the possibility that there is some contribution by SPMV RNA.

In summary, SPMV CP shares many features with other virus-encoded pathogenesis-associated proteins, but with an unusual capability of slowing the suppressive effects of PVX $\mathrm{p} 25$. From this and other studies, it is evident that multifunctional plant virus proteins such as SPMV CP play important biological and biochemical roles that affect disease outcomes in the plant cell. The results from this study reinforce the supposition that the effect of a virus-encoded pathogenesis-associated protein on gene silencing is dependent on the expression system and the type of gene silencing inducer, and is not necessarily coupled with-or a saltatory step toward-the suppression of gene silencing.

\section{MATERIALS AND METHODS}

\section{Recombinant PVX and TBSV plasmids.}

The PVX vector pPC2S (Chapman et al. 1992) was modified to abolish the second SalI site at nucleotide 6572, creating a new PVX derivative, PVX-E1S. The plasmid pSPMV-1 containing the full-length wild-type SPMV cDNA (Turina et al. 1998) was digested with SpeI and BsrGI and treated with DNA polymerase I large fragment (Klenow enzyme) to excise the SPMV CP gene. This blunt-ended fragment (nucleotides 63 to 709) was inserted in both directions at the EcoRV site (nucleotide 5677) of pPVX-E1S to create PVX-SPCP(+) and PVX-SPCP(-), with the SPMV CP in the plus- and minussense orientation, respectively (Fig. 1A). Approximately 0.5 $\mu \mathrm{g}$ of SpeI-linearized PVX cDNA template was used for in vitro RNA transcription with cap analog (Scholthof et al. 1995a).

The generation of recombinant TBSV-based plasmids DI-P and pTG were described previously (Qiu et al. 2002). Briefly, pTG was similar to the constructs in Figure 2A, but the GFP gene was substituted for the SPMV CP gene. The DI-P construct is a TBSV DI-based gene vector with the $3^{\prime}$ third of the GFP gene; it is used as a dual component with TBSV to evaluate either replication, silencing, or both. The SPMV coding region was amplified from pSPMV-1 (Turina et al. 1998) with the forward primer SPMVCPXhoI (5'-GGCTCGAGACAC ATGGCTCCTAAGCGTTCCAGG-3'; the XhoI site is underlined, and the start codon, ATG on the cDNA, is in bold) and the reverse primer SPMVCPSacI (5'-GGGAGCTCTTATGAA GACTGAAGCTCGCTAGGCGCCAG-3'; the SacI site is underlined). The SPMV CP coding region was inserted into a plasmid $\mathrm{pKAN}-2$ to generate an intermediate recombinant plasmid pKAN-2-SPCP. To generate plasmid TBSV-SPCP(+), 
the SPMV CP coding region was removed from the pKAN-2SPCP by digestion with $\mathrm{XhoI}$ and $\mathrm{SacI}$ restriction enzymes and cloned in the plus-sense orientation, into the pHST-2-14 vector that was digested with the same enzymes. To generate plasmid pTBSV-SPCP(-), with the SPMV CP ORF in the minus orientation, a SPMV fragment covering the SPMV CP coding region was removed from the pSPMV-1 with SpeI and BsrGI restriction enzymes, treated with Klenow enzyme, and then inserted into the Klenow-treated BglII site of the TBSV-BglII vector that has a $B g l$ II site in the multiple cloning site of pHST-2-14 (Scholthof 1999a). The TBSV-BglII vector was created by deleting a modified GFP (sGFP) gene in the plasmid pTBSV-sGFP with $B g l \mathrm{II}$ digestion, followed by religation. The plasmid TBSV-sGFP has two BglII sites bordering the sGFP gene (Sit et al. 1998). PVX- and TBSV-derived transcripts were inoculated onto plants at the three-leaf stage (Scholthof et al. 1995a). All plants were maintained in a growth chamber $\left(24^{\circ} \mathrm{C}, 14-\mathrm{h}\right.$ light; $20^{\circ} \mathrm{C}, 10$-h dark).

\section{GFP transgenic plants.}

GFP transgenic $N$. benthamiana line 16C (NbGFP) and Agrobacterium tumefaciens $\mathrm{C} 58 \mathrm{C} 1$ (pCH32) containing the binary vector $\mathrm{pBin}-35 \mathrm{~S}-\mathrm{GFP}$ (Haseloff et al. 1997) referred to as $\mathrm{Agro} / \mathrm{GFP}$ in this manuscript, were generously provided by D. C. Baulcombe.

\section{Plasmids for agroinfiltration.}

The SPMV CP coding region was excised from a full-length SPMV cDNA (pSPMV1) (Turina et al. 1998) with SpeI and BsrGI. This fragment was treated with DNA polymerase I large fragment (Klenow enzyme) and cloned into the SmaI site of pKAN in both orientations to create pK-SPMVCP(+) and pK-SPMVCP(-). These intermediate plasmids were digested with $X b a \mathrm{I}$ and $S a c \mathrm{I}$ and the resultant fragments containing the SPMV CP coding regions were ligated to the XbaI-SacI sites of pGPTV (Becker et al. 1992) to generate plasmids pG$\operatorname{SPCP}(+)$ and pG-SPCP(-).

The constructs subsequently were transferred into A. tumefaciens EHA105 (Hood et al. 1993) by triparental mating (Ditta et al. 1980). The resultant Agrobacterium transformants, Agro/SPCP(+) and Agro/SPCP(-), were grown overnight, collected, and resuspended in a solution of $10 \mathrm{mM} 2-[\mathrm{N}-\mathrm{mor}-$ pholine]ethanesulfonic acid, $10 \mathrm{mM} \mathrm{MgCl}_{2}$, and $150 \mu \mathrm{M}$ acetosyringone to a cell density of 0.5 at $600 \mathrm{~nm}$. The cultures then were incubated at room temperature for $2 \mathrm{~h}$ prior to infiltration into wild-type or NbGFP $N$. benthamiana leaves (English et al. 1997). For the PVX-SPMV-CP constructs, at 1 or 14 days following inoculation, the plants were infiltrated with Agro/GFP (English et al. 1997).

\section{Fluorescence imaging.}

Visual observation of the fluorescence transition on the leaves was made with a 100-W long-wave UV lamp. Photographs were made with Kodak Ektachrome Panther 400 ASA film and a yellow filter, with a 1-s exposure at f/5.6.

\section{RNA and protein analysis.}

Total RNA was extracted from systemically infected leaves and assayed for the presence of viral RNAs using SPMV- or PVX-specific probes as previously described (Scholthof et al. 1995b; Scholthof 1999b). Total plant RNAs for the detection of GFP mRNA accumulation was isolated from leaf tissues by a method modified from a hot-phenol procedure (Verwoerd et al. 1989). The subsequent RNA fractionation, blotting, and hybridization with ${ }^{32} \mathrm{P}-\mathrm{dCTP}$-labeled GFP-specific probes that were made by a random-priming method were performed as previously described (Qiu et al. 2002). Plasmid pEA-2 con- taining 28S rRNA cDNA derived from sugar beet leaves was used to monitor total RNA (Batten et al. 2000).

Immunoblot assays were carried out to analyze the expression of SPMV CP by PVX-SPCP(+). Approximately $0.4 \mathrm{~g}$ of upper, noninoculated leaf tissues from $N$. benthamiana plants at $21 \mathrm{dpi}$ with recombinant PVX viruses were collected and ground in $200 \mu \mathrm{l}$ of $1 \times$ Tris-EDTA buffer $(10 \mathrm{mM}$ Tris, $1 \mathrm{mM}$ EDTA, pH 7.4). To detect SPMV CP, total plant proteins were extracted from $200 \mathrm{mg}$ of TBSV-SPCP(+)-inoculated leaves, electrophoresed through $12.5 \%$ sodium dodecyl sulfate-polyacrylamide gels using Tris-tricine buffer system, blotted, and incubated with polyclonal rabbit antisera specific to SPMV CP (Scholthof 1999b) or PVX CP (Agdia, Inc., Elkhart, IN), as previously described (Scholthof 1999b; Scholthof et al. 1995c).

\section{ACKNOWLEDGMENTS}

This research was funded by the Southwest Missouri State University Faculty Research Foundation (W. Qiu) and USDA-NRI grants (96-353033714 and 99-35303-7974) awarded to K.-B. G. Scholthof. We thank H. Scholthof and R. Omarov for their critical reviews and insightful comments, D. Baulcombe for the generous gifts of $N$. benthamiana $16 \mathrm{C}$ seed and the PVX vector, T. Sit for the pTBSV-sGFP plasmid, H. Hou for technical assistance, and L. Kovacs for helpful advice on constructing Agro/SPCP(+) and Agro/SPCP(-).

\section{LITERATURE CITED}

Anandalakshmi, R., Pruss, G. J., Ge, X., Marathe, R., Mallory, A. C. Smith, T. H., and Vance, V. B. 1998. A viral suppressor of gene silencing in plants. Proc. Natl. Acad. Sci. USA 95:13079-13084.

Batten, J. S., Scholthof, K.-B. G., Miller, M. E., and Martyn, R. D. 2000. cDNA probes for detection of specific dsRNAs from the fungal pathogen, Monosporascus cannonballus. J. Virol. Methods 84:209-215.

Becker, D., Kemper, E., Schell, J., and Masterson, R. 1992. New plant binary vectors with selectable markers located proximal to the left TDNA border. Plant Mol. Biol. 20:1195-1197.

Beclin, C., Berthome, R., Palauqui, J.-C., Tepfer, M., and Vaucheret, H. 1998. Infection of tobacco or Arabidopsis plants by CMV counteracts systemic post-transcriptional silencing of non-viral (trans)genes. Virology 252:313-317.

Bonneau, C., Brugidou, C., Chen, L., Beachy, R. N., and Fauquet, C. 1998. Expression of the rice yellow mottle virus P1 protein in vitro and in vivo and its involvement in virus spread. Virology 244:79-86.

Brigneti, G., Voinnet, O., Li, W.-X., Ji, L.-H., Ding, S.-W., and Baulcombe, D. C. 1998. Viral pathogenicity determinants are suppressors of transgene silencing in Nicotiana benthamiana. EMBO (Eur. Mol. Biol. Organ.) J. 17:6739-6746.

Bringloe, D. H., Gultyaev, A. P., Pelpel, M., Pleij, C. W. A., and Coutts, R. H. A. 1998. The nucleotide sequence of satellite tobacco necrosis virus strain $\mathrm{C}$ and helper-assisted replication of wild-type and mutant clones of the virus. J. Gen. Virol. 79:1539-1546.

Cabrera, O., and K.-B. G. Scholthof. 1999. The complex viral etiology of St. Augustine decline. Plant Dis. 83:902-904.

Chapman, S., Kavanagh, T., and Baulcombe, D. 1992. Potato virus X as a vector for gene expression in plants. Plant J. 2:549-557.

Culver, J. N., Lindbeck, A. G. C., and Dawson, W. O. 1991. Virus-host interactions: Induction of chlorotic and necrotic responses in plants by tobamoviruses. Annu. Rev. Phytopathol. 29:193-217.

Desvoyes, B., and Scholthof, K.-B. G. 2000. RNA:protein interactions associated with satellites of panicum mosaic virus. FEBS (Fed. Eur. Biochem. Soc.) Lett. 485:25-28.

Ding, S.-W., Shi, B.-J., Li, W.-X., and Symons, R. H. 1996. An interspecies hybrid RNA virus is significantly more virulent than either parental virus. Proc. Natl. Acad. Sci. USA 93:7470-7474.

Ditta, G., Stanfield, S., Corbin, D., and Helinski, D. R. 1980. Broad host range DNA cloning system for Gram-negative bacteria: Construction of a gene bank of Rhizobium meliloti. Proc. Natl. Acad. Sci. USA 77:7347-7351

English, J. J., Davenport, G. F., Elmayan, T., Vaucheret, H., and Baulcombe, D. C. 1997. Requirement of sense transcription for homology-dependent virus resistance and trans-inactivation. Plant J. 12:597-603.

Gilchrist, D. G. 1998. Programmed cell death in plant disease: the purpose and promise of cellular suicide. Annu. Rev. Phytopathol. 36:393-414. 
Goldberg, K.-B., Kiernan, J., and Shepherd, R. J. 1991. A disease syndrome associated with expression of gene VI of caulimoviruses may be a nonhost reaction. Mol. Plant-Microbe Interact. 4:182-189.

Greenberg, J. T. 1997. Programmed cell death in plant-pathogen interactions. Annu. Rev. Plant Physiol. Plant Mol. Biol. 48:525-545.

Haseloff, J., Siemering, K. R., Prasher, D. C., and Hodge, S. 1997. Removal of a cryptic intron and subcellular localization of green fluorescent protein are required to mark transgenic Arabidopsis plants brightly. Proc. Natl. Acad. Sci. USA 94:2122-2127.

Heath, M. C. 2000. Nonhost resistance and nonspecific plant defenses Curr. Opin. Plant Biol. 3:315-319.

Hood, E. E., Gelvin, S. B., Melchers, L. S., and Hoekema, A. 1993. New Agrobacterium helper plasmids for gene transfer to plants. Trans. Res. 2:208-218.

Kasschau, K. D., and Carrington, J. C. 1998. A counterdefensive strategy of plant viruses: suppression of posttranscriptional gene silencing. Cell 95:461-470.

Pruss, G., Ge, X., Shi, X. M., Carrington, J. C., and Vance, V. B. 1997. Plant viral synergism: the potyviral genome encodes a broad-range pathogenicity enhancer that transactivates replication of heterologous viruses. Plant Cell 9:859-868.

Qiu, W., Park, J.-W., and Scholthof, H. B. 2002. Tombusvirus P19-mediated suppression of virus-induced gene silencing is controlled by genetic and dosage features that influence pathogenicity. Mol. PlantMicrobe Interact. 15:269-280.

Qiu, W. P., and Scholthof, K.-B. G. 2000. In vitro- and in vivo-generated defective RNAs of satellite panicum mosaic virus define cis-acting RNA elements required for replication and movement. J. Virol. 74:2247-2254.

Qiu, W. P., and Scholthof, K.-B. G. 2001a. Defective interfering RNAs of a satellite virus. J. Virol. 75:5429-5432.

Qiu, W. P., and Scholthof, K.-B. G. 2001b. Genetic identification of multiple biological roles associated with the capsid protein of satellite panicum mosaic virus. Mol. Plant-Microbe Interact. 14:21-30.

Routh, G., Ngon A Yassi, M., Rao, A. L. N., Mirkov, T. E., and Dodds, J. A. 1997. Replication of wild-type and mutant clones of satellite tobacco mosaic virus in Nicotiana benthamiana protoplasts. J. Gen. Virol. 78:1271-1275.

Schoelz, J. E., Goldberg, K.-B., and Kiernan, J. 1991. Expression of cauliflower mosaic virus (CaMV) gene VI in transgenic Nicotiana bigelovii complements a strain of CaMV defective in long-distance movement in nontransformed $N$. bigelovii. Mol. Plant-Microbe Interact. 4:350-355.

Scholthof, H. B. 1999a. Rapid delivery of foreign genes into plants by direct rub-inoculation with intact plasmid DNA of a tomato bushy stunt virus gene vector. J. Virol. 73:7823-7829.

Scholthof, K.-B. G. 1999b. A synergism induced by satellite panicum mosaic virus. Mol. Plant-Microbe Interact. 12:163-166.

Scholthof, H. B., Scholthof, K.-B. G., and Jackson, A. O. (1995a). Identification of tomato bushy stunt virus host-specific symptom determinants by expression of individual genes from a potato virus $\mathrm{X}$ vector Plant Cell 7:1157-1172.

Scholthof, H. B., Scholthof, K.-B. G., Kikkert, M., and Jackson, A. O. (1995b). Tomato bushy stunt virus spread is regulated by two nested genes that function in cell-to-cell movement and host-dependent systemic invasion. Virology 213:425-438.

Scholthof, K.-B. G., Scholthof, H. B., and Jackson, A. O. 1995c. The tomato bushy stunt virus replicase proteins are coordinately expressed and membrane associated. Virology 208:365-369.

Sit, T. L., Vaewhongs, A. A., and Lommel, S. A. 1998. RNA-mediated trans-activation of transcription from a viral RNA. Science 281:829832.

Toth, R. L., Chapman, S., Carr, F., and Santa Cruz, S. 2001. A novel strategy for the expression of foreign genes from plant virus vectors. FEBS (Fed. Eur. Biochem. Soc.) Lett. 489:215-219

Turina, M., Desvoyes, B., and Scholthof, K.-B. G. 2000. A gene cluster encoded by panicum mosaic virus is associated with virus movement. Virology 266:120-128.

Turina, M., Maruoka, M., Monis, J., Jackson, A. O., and Scholthof, K.-B. G. 1998. Nucleotide sequence and infectivity of a full-length cDNA clone of panicum mosaic virus. Virology 241:141-155.

Verwoerd, T. C., Dekker, B. M. M., and Hoekema, A. 1989. A small-scale procedure for the rapid isolation of plant RNAs. Nucleic Acids Res. $17: 2362$.

Voinnet, O., Lederer, C., and Baulcombe, D. C. 2000. A viral movement protein prevents spread of the gene silencing signal in Nicotiana benthamiana. Cell 103:157-167.

Voinnet, O., Pinto, Y. M., and Baulcombe, D. C. 1999. Suppression of gene silencing: A general strategy used by diverse DNA and RNA viruses of plants. Proc. Natl. Acad. Sci. USA 96:14147-14152. 